\title{
A Novel Approach to Optimization Problem without Objective Function
}

\author{
Yingying $\mathrm{Su}^{1}$ Wenjin $\mathrm{Hu}^{2}$ Ling $\mathrm{Nie}^{2}$ Wen $\mathrm{Ye}^{2}$ Taifu $\mathrm{Li}^{2}$ \\ ${ }^{1}$ Dept. of Electronic Information and Automation, Chongqing Inst. of Tech., Chongqing 400050, P. R. China; \\ ${ }^{2}$ Dept. of Electronic Information Eng., Chongqing Univ. of Sci. and Tech., Chongqing 400050, P. R. China
}

\begin{abstract}
For optimization problem in complex systems, normally, the objective funtion is hardly obtained or quantified. In this paper, a novel approach is presented. Its first procedure is to model the objective function by fitting complete data with NN. Secondly, global optimization solutions would be searched for the fitted objective function with genetic algorithm (GA). Moreover, Peaks function inside MATLAB and actual PID control system were selected to demostrate the approach, respectively. The results show that the optimal values and the correspnding solutions between original function and fitted function were both very close. Therefore, the methodology, which combines modeling approach NN with global optimization algorithm GA, could effectively solve optimization problem without objective function.
\end{abstract}

Keywords: Optimization, Modeling, Neural network, Genetic algorithm

\section{Introduction}

Optimization method is not only a branch of applied mathematics, and also a main method of modern engineering design. It brings in obvious economic benefits, technique value and management profits. Therefore, it has attracted more and more attentions from academic community and engineering field. Its mathematic form is conventionally given by

$$
\begin{array}{ll}
\min & (\text { or max }) \quad f\left(x_{1}, x_{2}, \cdots, x_{n}\right) \\
\text { s.t. } & \mathbf{c}\left(x_{1}, x_{2}, \cdots, x_{n}\right) \leq 0 \\
& \mathbf{c e q}\left(x_{1}, x_{2}, \cdots, x_{n}\right)=0 \\
& \mathbf{A} \cdot \mathbf{x} \leq \mathbf{b} \\
& \text { Aeq } \cdot \mathbf{x}=\mathbf{b e q} \\
& \mathbf{l b} \leq \mathbf{x} \leq \mathbf{u b}
\end{array}
$$

The optimization problems will be simply solved when the objective function is certain. However, in fact the objective function like formula (1) is hardly obtained for its complex nonlinear connection with influencing parameters. To this problem, the extensively adopted method is experiment design. It makes use of the regression or variance analysis to determine the optimal parameters after the uniform design or orthogonal experiment [1]. However, there are still several shortcomings: (1) The hardship data analysis after the experiment, (2) The poor adaptability of traditional regression analysis and variance analysis to multi-input/multi-output problems, (3) The difficulty in searching the global optimal parameter combination.

In this aspect, the black box modeling methods with fitting input and output data were once used to reconstruct the objective functions. Neural network (NN) [2][3] is one of main methods, and has already been applied in material science and technology [4][5]. Yet, the universal optimization methodology without objective function hasn't been presented. The studies only remain in a stage of solving some physical cases. For this, in this paper, authors presented modeling and optimization methodology without objective function. It firstly approaches the nonlinear mapping from influential parameters to objective performance, obtaining the smooth super-surface with computational intelligence such as fuzzy logic (FL), NN, support vector machine (SVM) and so on. Secondly, genetic algorithm (GA) or immune optimization algorithm (IA) can be effectively utilized to search the global optimal with this fitting objective function. In this paper, the modeling and optimization methodology for complex system without objective function will be carried out with NN and GA for instance.

\section{Modeling in complex systems based on NN}

NN can emulate the physical structure as well as the memory function of mankind brain. It has been testified as a universal function approximator on mathematics, and can approximate every nonlinear function in $\mathrm{L}^{2}$ norm [6]. In this way, it is an effectively method in solving the function modeling problems, where the characteristic pattern is implicit. In hundreds of NN 
types, multi-layer feedforward NN in Fig.1 is one of the widely adopted models.

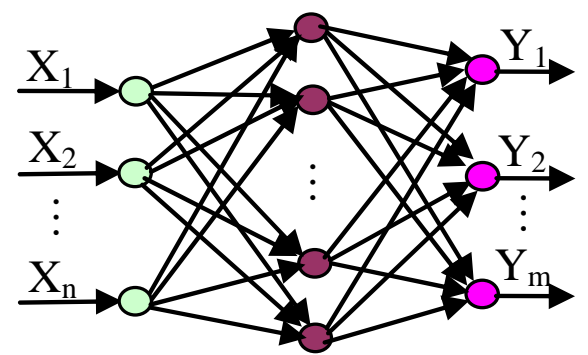

Fig.1: The structure of multi-layer feedforward NN.

Usually, multi-layer feedforward NN adopts Back Propagation (BP) as learning algorithm. Its learning procedure is as following:

1) Confirm the topological structure and the learning parameters of $\mathrm{NN}$; $\mathrm{NN}$;

2) Initialize the weight and threshold values of

3) Obtain the training data: input vector $X_{p}$ and target vector $Y_{p}$;

4) Compute the state of hidden layer neurons and the actual output of NN, (Sigmoid function is supposed as the active function);

$$
\begin{aligned}
O_{p k} & =f_{k}\left(\sum W_{k j} O_{j}-\theta_{k}\right) \\
& =\frac{1}{1+\exp \left[-\left(\sum W_{k j} O_{j}-\theta_{k}\right)\right]}
\end{aligned}
$$

5) Compute the value of error energy function;

$$
E_{p}=\frac{1}{2} \sum_{k=1}^{n}\left(Y_{p k}-O_{p k}\right)^{2}
$$

6) Compute the training error of current $\mathrm{NN}$ : output-layer:

$$
\delta_{p k}=O_{p k}\left(1-O_{p k}\right)\left(Y_{p k}-O_{p k}\right)
$$

hidden layer:

$$
\delta_{p j}=O_{p j}\left(1-O_{p j}\right) \sum_{k} \delta_{p k} W_{k j}
$$

7) Adjust weight and threshold values:

$$
\begin{aligned}
W_{j i}(n+1) & =W_{j i}(n)+\eta \delta_{p j} O_{p j} \\
& +\alpha\left(W_{j i}(n)-W_{j i}(n-1)\right) \\
\theta_{j}(n+1)= & \theta_{j}(n)+\eta \delta_{p j} \\
+ & \alpha\left(\theta_{j}(n)-\theta_{j}(n-1)\right)
\end{aligned}
$$

8) Turn to step 3), or stop at the stopping epoch, or the error energy function has been satisfied.

Here, selecting Peaks function inside MATLAB as approaching example, the approximating ability of NN will be tested. The surface of original Peaks function is shown in Fig.2, and its mathematic expression is given in equation (9):

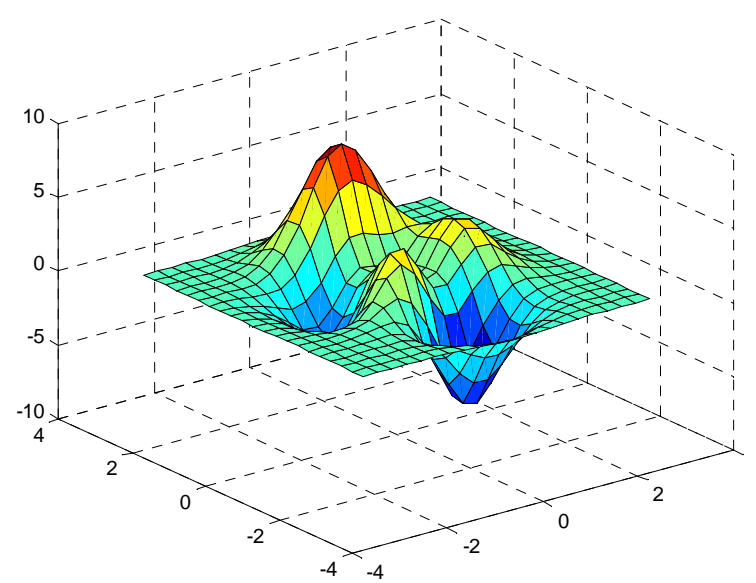

Fig.2: The surface curve of original Peaks function.

$$
\begin{aligned}
z= & \operatorname{Peaks}(x, y) \\
= & 3(1-x)^{2} \cdot e^{\left[-x^{2}-(y+1)^{2}\right]} \\
& -10\left(x / 5-x^{3}-y^{5}\right) \cdot e^{\left(-x^{2}-y^{2}\right)} \\
& -\frac{1}{3} e^{\left[-(x+1)^{2}-y^{2}\right]}
\end{aligned}
$$

In MATLAB NN Tools, a multi-layer feedforward NN is confirmed as 2-22-1 in Fig.3. Sigmoid and pure functions are selected as the transformation function for hidden layer and output-layer, respectively. 441 data pairs of input $\mathrm{X}, \mathrm{Y}$ with interval [-3:0.3:3] and the corresponding value from equation (3) are selected as the training data. Then $\mathrm{NN}$ is trained with gradient descend method Levenberg-Marquardt algorithm. Finally, the surface of fitted Peaks function is obtained in Fig.4. Linear relation between output of original Peaks function and that of fitted Peaks function with the 441 data pairs are shown in Fig. 5.

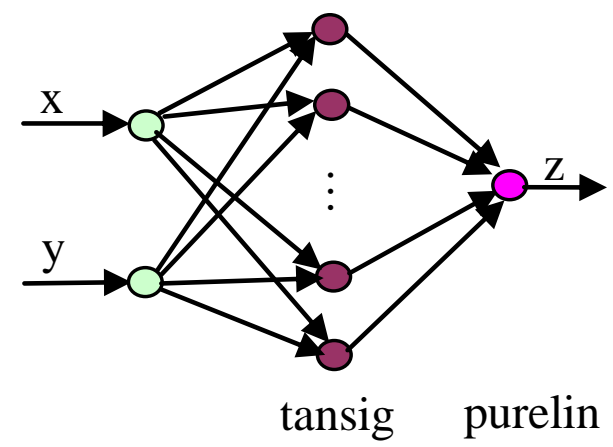

Fig.3: The structure of NN for fitting Peaks function 


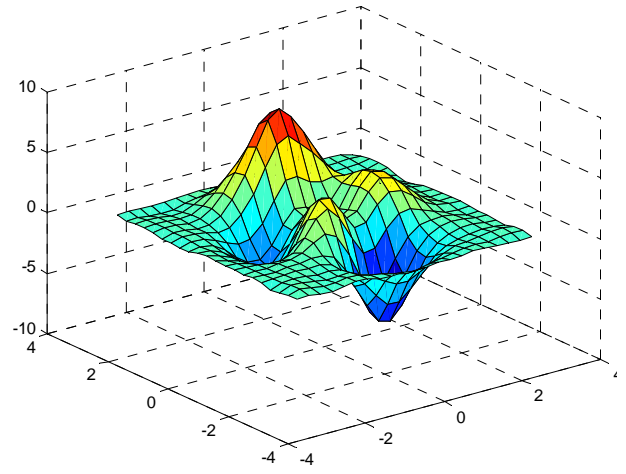

Fig.4: The surface of fitted peaks function with NN

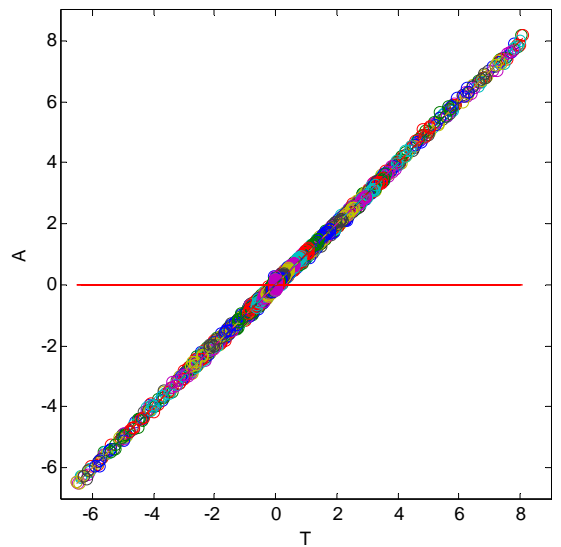

Fig.5: Linear relation between peaks function and fitted peaks function

The best linear fitting equation in Fig.5 is

$A=0.996 T+0.00131$

where, $\mathrm{T}$ represents the output of original peaks function and A represents the output of fitted peaks function with NN. It shows that NN has excellent nonlinear approximating capability.

\section{Global optimization with GA for approaching model}

In order to further verify the idea above-mentioned, two optimal problems should be discussed, one is (11): min Peaks $(x, y)$

$$
\text { s.t. }-3 \leq x, y \leq 3
$$

where Peaks $(x, y)$ is original peaks function with respect to $x, y$. Another is fitted peaks function given in equation (12):

$$
\begin{aligned}
& \min \quad N_{-} \operatorname{Peaks}(x, y) \\
& \text { s.t. }-3 \leq x, y \leq 3
\end{aligned}
$$

where $N_{\text {_ }} \operatorname{Peaks}(x, y)$ is fitted peaks function also related to $x, y$.

Firstly, in MATLAB GA Toolbox, optimize the problem (11). Parameters in the software are settled as populations 40 , length of binary encoding 25 , crossover probability 0.7 and mutation probability $0.7 /(25 \times 2)=$ 0.014 . With evolution of 50 generations, the optimal value and the corresponding solutions are obtained as $z_{1}=-6.5511,\left(x_{1}, y_{1}\right)=(0.2283,-1.6255)$. And then, in order to enhance the accuracy, they are further searched in MATLAB Optimization Toolbox when selecting $\left(x_{1}, y_{1}\right)$ as initial point. The final value and solutions are $z_{2}=-6.5511, \quad\left(x_{2}, y_{2}\right)=(0.2283,-1.6256)$.

In the similar way, with MATLAB GA Toolbox, the problem (12) is also optimized at 50 generations evolution. The populations in original generation, 20 generations and 40 generations are respectively shown in Fig.6 (a), (b) and (c). The changing curves of best fitness and average fitness are shown in Fig.7. Here, the optimal value and solutions of problem (12) are acquired as $z_{3}=-6.5057,\left(x_{3}, y_{3}\right)=(0.2402,-1.6462)$.

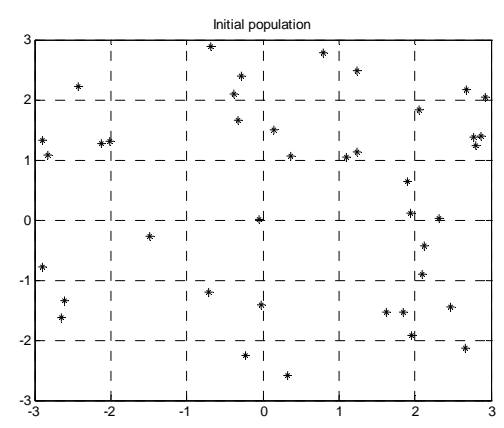

(a)

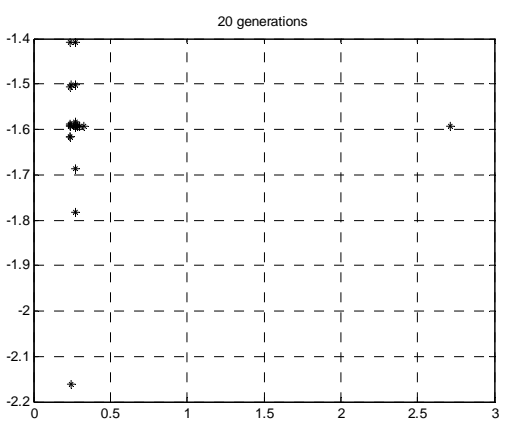

(b)

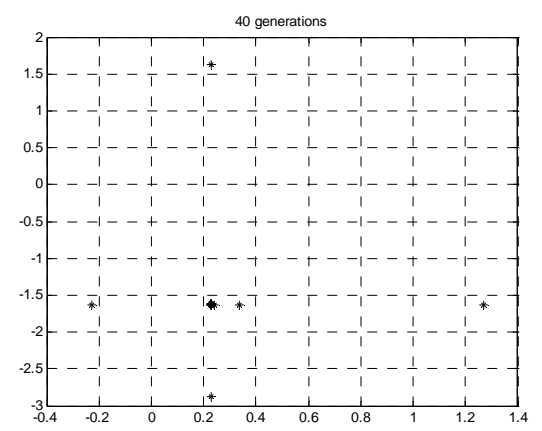

(c)

Fig.6: The population distribution changing with evolution 


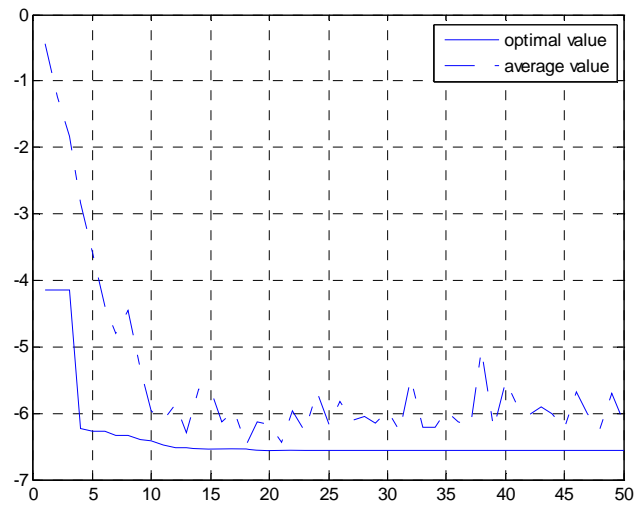

Fig.7: The best fitness and average fitness changing with evolution

Analyzing the optimization results of problem (11) and (12), the optimal value $\mathrm{z} 2, \mathrm{z} 3$ and the optimal solutions are all extremely similar. It has verified that NN can effectively approximate nonlinear continuous functions. On the basis, the optimal value and parameters can be accurately acquired by globally searching algorithm GA.

\section{Application}

In the section 2 and 3, the method above-mentioned is verified with simulation. In fact, it was also used to optimize the comprehensive performance in analog PID control system. Since comprehensive performance in analog PID control systems is usually determined by various indices, such as dynamic indices of overshoot $\sigma$, rising time $t_{r}$, setting time $t_{s}$, and steady state indices of steady state error $e_{s s}$, etc, it results in the difficulty of modeling between various performance indices and influencing parameters proportion $K_{p}$, integration $K_{i}$, derivative $K_{d}$. However, a skillful expert can easily evaluate the comprehensive control quality according to the response curve. In this way, the comprehensive performance can be quantified by Expert Knowledge (EK). Then NN can approach the smooth super-surface between expert evaluation and influencing parameters. On the basis of object function modeling, GA is able to optimize the global comprehensive performance.

With this method, a PID control system is optimized and the final optimal parameters are $R_{P}=1.02 \times 10^{6} \Omega, R_{d}=8.54 \times 10^{4} \Omega, C_{i}=20 \mu \mathrm{F}$. The corresponding response curve is shown in Fig.8. Compared with the optimal value and solutions in Uniform Design, the overshoot and setting time here are both smaller in Table 1 . Therefore, the optimal tuning parameters of PID control system are effectively obtained with the method in the paper.

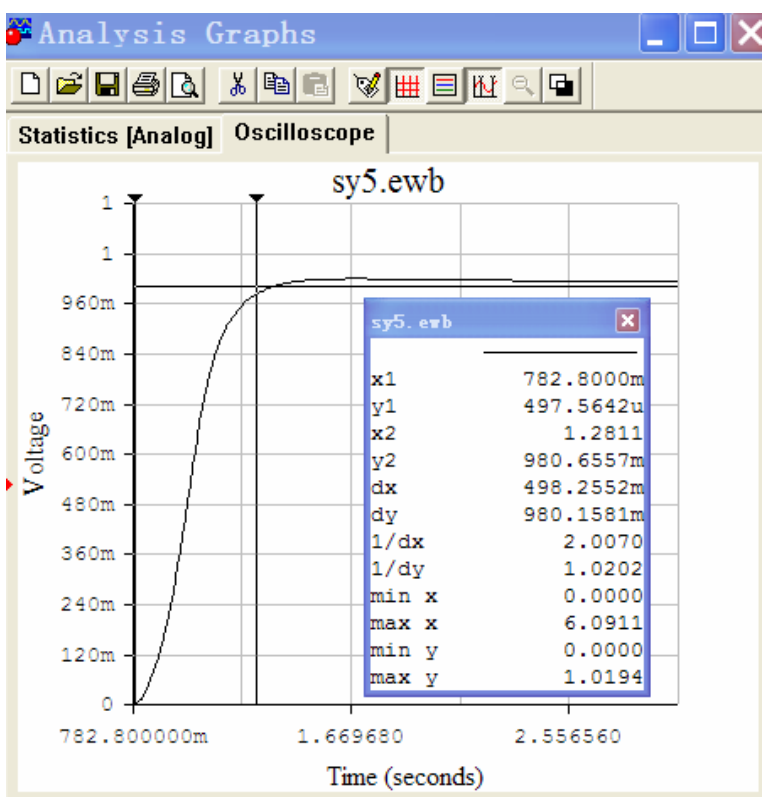

Fig.8: The optimal response of PID control system based on NN, EK and GA

\begin{tabular}{|c|c|c|c|}
\hline \multicolumn{2}{|c|}{$\begin{array}{c}\text { Optimization } \\
\text { with Uniform Design }\end{array}$} & \multicolumn{2}{|c|}{$\begin{array}{c}\text { Optimization } \\
\text { with method in the paper }\end{array}$} \\
\hline $\begin{array}{l}\text { Decision-making } \\
\text { variables }\end{array}$ & $\begin{array}{c}\text { Performance } \\
\text { index }\end{array}$ & $\begin{array}{c}\text { Decision-making } \\
\text { variables }\end{array}$ & $\begin{array}{c}\text { Performance } \\
\text { index }\end{array}$ \\
\hline $\begin{array}{ccc}R_{p} & C_{i} & R_{d} \\
\left(10^{6} \Omega\right) & (\mathrm{uF}) & (10 \Omega)\end{array}$ & $\begin{array}{cc}\sigma & t_{s} \\
(\%) & (\mathrm{s})\end{array}$ & $\begin{array}{ccc}R_{p} & C_{i} & R_{d} \\
\left(10^{6} \Omega\right) & (\mathrm{uF}) & (10 \Omega)\end{array}$ & $\begin{array}{c}\sigma \\
(\%)\end{array}$ \\
\hline $\begin{array}{lll}1.2 & 16 & 8 \\
\end{array}$ & 0.61 & $\begin{array}{lll}1.02 & 20 & 8.54\end{array}$ & 0.50 \\
\hline
\end{tabular}

Table1: The optimal results compared with the method in the paper and that in Uniform Design

\section{Conclusions}

In thousands of mechanical products and systems, there are usually short of mathematic functions between performance and influencing parameters. For many material composing puzzles, it also makes difficulties in performance modeling related to ingredient percents. In addition, in lots of production process, it is even hard to obtain quality modeling with respect to technical parameters. Aimed at these problems, the study abovementioned can model the objective-function to solve this typical of optimization puzzle. And then, GA can be effectively applied for optimizing global performance and searching optimal decision-making variables.

\section{Acknowledgement}

The authors gratefully acknowledge the support of Chongqing Educational Committee Sci. \& Tech. Research Project (Grant No. KJ071411).

\section{References}


[1] Y. Wang and K. Fang, Uniform distribution and experiment design, Cinese Science Bulletin, 26: 65-70, 1981.

[2] M. R. Warnes, J. Glassley and G. A. Montague. On data-based modeling techniques for fermenation process, Processes Biochem, 31: 147-155, 1996.

[3] F. Shi and J.G. Lu, Fuzzy optimization of cold extrusion mold based on genetic algorithm and neural network, Chinese Journal of Mechanical Engineering, 38: 45-49, 2002.

[4] J.X. Qiu, S.H. Zhang and G.L. Li, Application of combining uniform design, neural network and genetic algorithm to optimize process parameters of internal high pressure forming, Journal of Plasticity Engineering, 12 : 76-79, 2005.

[5] Z.B. Gu, H.J. Li, K.Z Li and A.J. Li, Modeling of isothermal CVI process of $\mathrm{C} / \mathrm{C}$ composites by fuzzy Neural Network, Raremetal Materials and Engineering, 33: 1037-1040, 2004.

[6] T.F. Li, Y.Y. Su and B.X. Zhong, Remodeling for fuzzy PID controller based on neural networks, Advance in soft computing, Springer-verlag, 714725, 2007. 